\title{
Effects of a personalized home-based training program among patients suffering from Marfan syndrome: a pilot randomized and controlled study
}

\author{
Steeve Jouini ${ }^{1}$, Olivier Milleron ${ }^{2,3,4}$, Ludivine Eliahou ${ }^{3,4}$, Guillaume Jondeau ${ }^{2,3,4}$, \\ Damien Vitiello, ${ }^{1, *}$ \\ ${ }^{1}$ Institute of Sport and Health Sciences of Paris, Universite de Paris, Paris, France; \\ ${ }^{2}$ Laboratory for Vascular Translational Science, Hôpital Bichat-Claude-Bernard, Université de Paris, Paris, France; \\ ${ }^{3}$ Centre national de référence pour le syndrome de Marfan et pathologies apparentés, Assistance Publique-Hôpitaux de Paris, Hôpital Bichat, Paris, \\ France; \\ ${ }^{4}$ Service de Cardiologie, Centre National de Référence Pour le Syndrome de Marfan et Apparentés, AP-HP, Hôpital Bichat, Paris, France.
}

SUMMARY Marfan syndrome (MFS) is an autosomal hereditary pathology affecting 1:5000 peoples. Alteration of the fibrillin 1 gene (FBN1) results in haplo-insufficiency of the FBN1 protein mainly altering the vascular system. International recommendations have gradually allowed MFS patients to perform training programs because of its potential benefits. However, to date, there are no data on the effect of a long training period in these patients. The aim of the present study is to investigate the effect of a 3-month personalized home-based training on quality of life (QoL) of patients suffering from MFS. At least 50 MFS patients were included in the study. They were randomly placed into 4 groups: control group; endurance; resistance and endurance + resistance training groups. The training program lasted 3 months and is performed at patients' home. There were 2 training sessions per week telemonitored by a specialist of physical activity and cardiology. Pre and post-training evaluations were performed at the Bichat-Paris Hospital, France. They consisted of assessing psychometrics based on self-administered questionnaires (FiRST, GPAQ, ISP-25, MOS SF-36) and physiological parameters such as the peak oxygen consumption, aorta diameter, cardiac ventricle function and skeletal muscle power at rest and during exercise. Our preliminary results showed an improvement of 50\% in QoL, cardiorespiratory fitness and skeletal muscle power in a patient who completed the combined training program. This experimental approach might be a new alternative way for MFS patients' care that may improve their QoL, cardiorespiratory fitness and skeletal muscle power.

Keywords Marfan, telerehabilitation, personalized training

\section{Introduction}

Marfan syndrome (MFS) is an autosomal dominant genetic pathology affecting the cardiovascular system, the respiratory system, skeletal muscles, bones and eyes. This syndrome is the result of a mutation in the gene encoding fibrillin type I protein (FBN1), which forms a microfibrillar network interacting with elastin fibers to form the standard extracellular matrix.

In MFS patients, the most serious risk is a progression to aortic dissection, which is associated with a high rate of mortality (i.e. 1\% per hour during the first 48 hours). Several studies have highlighted anomalies in the left ventricle (LV) size, function, myocardial deformation and myocardial fibrosis $(1,2)$. It has also been shown that the cause of LV dysfunction was also due to mutation of the FBN1 gene, related to the Transforming Growth Factor (TGF) pathway (3). Patients also have a slender morphotype with low muscle mass (4). In addition, they suffer from a decrease maximal quadriceps strength associated with a decrease in muscular mass (5). Musculoskeletal pains reduce endurance capacity as well ( 6 ).

All the aforementioned disorders are associated with pain and disability, which affect professional activity, leisure, and family life. Furthermore, aortic dilation and the associated risk of aortic dissection are a source of anxiety for these patients (Peters et al. 2001) and depression is often found in patients MFS (7). In addition, quality of life (QoL) assessed by the SF-36 questionnaire is very impaired in these patients (8), particularly in patients with the largest aortic dilations. 
Therefore, it is essential to find a new global approach that should be proposed to reduce the cardiovascular impact but also the psychological impact (7) of MFS.

Physical activity (PA) may play a vital role in secondary aortic dilation and dissection prevention in patients with MFS in association with routine clinical management based on prescription of treatment with beta-blockers and annual monitoring of aortic diameter (9). As reported by the new 2020 guidelines on sports cardiology and exercise in patients with cardiovascular disease of the European Society of Cardiology (ESC) (10) the prescription of regular physical activity is highly beneficial for patients with cardiovascular diseases and reduced their mortality. In patients with aortopathy, regular PA has a positive effect on hypertension and concomitant future risk of dissection. Moreover, regular PA appeared to be well tolerated in MFS patients and induced beneficial physical effects (11). However, to our knowledge, this is the only existing small prospective cohort study conducted in these patients and there are no randomized controlled trials or any prospective data regarding risks of more intensive PA practice in MFS patients. Finally, home-based training may represent a new alternative way for MFS patients' care. Indeed, it has been demonstrated that heart rate monitoring and the Internet were effective tools for management of patients after coronary artery disease by improving their cardiopulmonary fitness after a 12-week home-based program (12).

In this context, the present study aimed at determining the impact of a 3-month personalized homebased training on the QoL of MFS patients.

\section{Materials and Methods}

This study was designed as an interventional, monocentral, prospective and randomized controlled trial at the Marfan National Reference Center in the Hospital of Bichat, Paris, France. They were 3 phases during the study: Phase 1) baseline evaluations at the hospital; Phase 2) post 3-month home-based personalized training evaluations at the hospital; Phase 3) Phone call evaluations 3 months after the end of training. All patients involved in the study provided written informed consent before entering the study. The study complies with the Declaration of Helsinki. The institutional ethics committee approved the study protocol (\#2020-A0175138), the French Society of Cardiology promotes the study and informed consent will be obtained from the subjects.

\subsection{Population}

Patients with MFS aged between 18 and 65 years were included in the study. The patients had to receive an optimal and stable dose of ACE inhibitors and Betablockers. They also had to be able to realize physical exercise and to possess social security insurance. The contradictions for randomization in the study were: pregnant patients, patients with cardiovascular diseases not due to MFS, an aortic dissection, an aortic diameter > $45 \mathrm{~mm}$, an aortic regurgitation, a non-controlled resting hypertension (diastolic blood pressure $>90 \mathrm{mmHg}$ and systolic blood pressure $>140 \mathrm{mmHg}$ ), who were non reachable by phone, who participated in another research project, who refused or who were not able to sign the informed consent form. Participants eligible for research were randomized into 4 experimental groups: Group 1 (control: no training), Group 2 (aerobic training), Group 3 (muscle strengthening training) and Group 4 (endurance + resistance training). The $\mathrm{R}$ program, with envelopes opened when the patient was randomized generated a randomization list.

\subsection{Evaluations during visits 1 and 2}

Cardiovascular capacity at rest was assessed before and after the training program. Patients underwent the following evaluations: $i$ ) Electrocardiogram (ECG) (200S-Cardioline). ii) Resting blood pressure assessment using an automatic tensiometer $\left(\right.$ dynamap $\left.^{\circledR}\right)$. iii) A standard echocardiography and a tissue Doppler imaging were performed to assess the ejection fraction (EF) of the left ventricle (LV), its diastolic diameter (LVDD), the systolic interventricular septum thickness (IVSTs), the global systolic and diastolic function of the LV (i.e. S, E and A waves of the lateral and septal walls) and the right ventricle (RV) (i.e. S wave, TAPSE and E/A peak), a full aorta analysis (i.e. ring, Valsalva sinus, tubular aorta, arch, descending thoracic aorta, and abdominal aorta) and a measure of the vena cava diameters during inspiration and expiration (Vivid 9 Dimension ${ }^{\circledR}$ ultrasound apparatus-GE Healthcare). A 2D strain echocardiography was performed to assess the global systolic longitudinal strain (GLS) of the LV and the RV.

\subsection{Assessment of oxygen consumption $\left(\mathrm{VO}_{2}\right)$ before} and after the training program

Patients performed a $\mathrm{VO}_{2}$ test on an ergometric bike. This exercise intensity was increased by 10 watts every minute until patients reach a peak of $\mathrm{VO}_{2}$ or until voluntary cessation. The ventilatory threshold 1 (VT1), the oxygen absorption efficiency slope (OUES), the ratio ventilation (VE) on carbon dioxide production $\left(\mathrm{VCO}_{2}\right)\left(\mathrm{VE} / \mathrm{VCO}_{2}\right)$ as a prognostic value was measured and a spirometry performed to evaluate the following parameters: forced vital capacity (FVC), forced expiratory volume in one second (FEV1), FEV1/FVC and peak expiratory flow 75\% (PEF75).

\subsection{Assessment of heart rate and blood pressure}

Heart rate (HR) was monitored during the test. Two HR references were obtained, one at the VT1 (HRVT1) and 
one at the peak of $\left(\right.$ HRpeak $\left.\mathrm{VO}_{2}\right)$. HRVT1 $+10-20 \%$ was used for targeting the intensity of training sessions and HRPeak $\mathrm{VO}_{2}$ was the HR limit to be reached during training sessions. The systolic blood pressure (SBP) and the diastolic blood pressure (DBP) were also carried out during the test. If the SBP was $\leq 160 \mathrm{mmHg}$ for at least 20 minutes, then the patient was allowed to continue the research. If this SBP was $>160 \mathrm{mmHg}$ during exercise, then the patient was excluded from research $(11,13-15)$. In parallel, the rating of perceived exertion (RPE) was evaluated during the test (16).

\subsection{Muscular capacity evaluations}

Patients performed one countermovement jump (cm), one squat jump (cm), repeated jumps ( $\mathrm{nb} / \mathrm{min})$ and an evaluation of the one-repetition maximum (1RM) based on 3 consecutive squats. The muscular capacity evaluations consisted of the evaluation of the muscle power and the maximal force contraction using a linear encoder $\left(\right.$ Gymaware $\left.^{\circledR}\right)$.

After these muscular capacity evaluations, patients performed different types of muscle-building movements under supervision (i.e. step, dynamic squat, isometric squat, crunch, abdominal and low back sheathing, isometric contraction of the quadriceps (chair position) and concentric contractions of the biceps (dumbbells of $1-5 \mathrm{~kg}$ ) (Supplemental Figure S1, http://www.irdrjournal. com/action/getSupplementalData.php?ID=82).

\subsection{Body composition}

The body composition of MFS patients was measured using a bioimpedance scale (Tanita Body composition Analyzer BC-420MA). The bodyweight and the percent of fat mass $(\mathrm{BF} \%)$, of lean mass $(\mathrm{LM} \%)$ and of water were assessed. The body mass index (BMI) was calculated using the following formula: BMI $=\mathrm{kg} / \mathrm{m}^{2}$, where $\mathrm{kg}$ is a patient's weight in kilograms, and $\mathrm{m}^{2}$ is the patient's height in meters squared.

\subsection{Psychometric questionnaires}

The assessment of QoL, level of physical activity, pain and pre-workout self-esteem were given to all patients included in the study. QoL was assessed by the MOS SF36 (Medical Outcome Study Short Form - 36) (17) questionnaire. The GPAQ (Global Physical Activity Questionnaire) (18) evaluated daily physical activity in sixteen questions. The FiRST (Fibromyalgia Rapid Screening Tool) scale assessed pain of patients (19) and the ISP-25 (Inventaire du Soi Physique-25) questionnaire assessed self-esteem of patients (20).

\subsection{Personalized home-based training program}

The training program followed the last ESC guidelines recommendations for physical activity (10). All patients included in training groups performed a 3-month personalized home-based training program with 2 sessions per week (Figure 1). The prescribed intensity of exercise was based on recommendations made by the European and American learned societies (9,21). These sessions were performed according to the level of patients evaluated during the initial cardiopulmonary and muscle capacity tests.

\subsubsection{Endurance training (Group 2)}

Endurance training consisted of a training circuit (TC) to lead patients to reach a target HR intensity evaluated during visit 1 baseline evaluations (Table 1). The intervals of recovery times, efforts, and number of series were adapted according to aerobic capacity of the

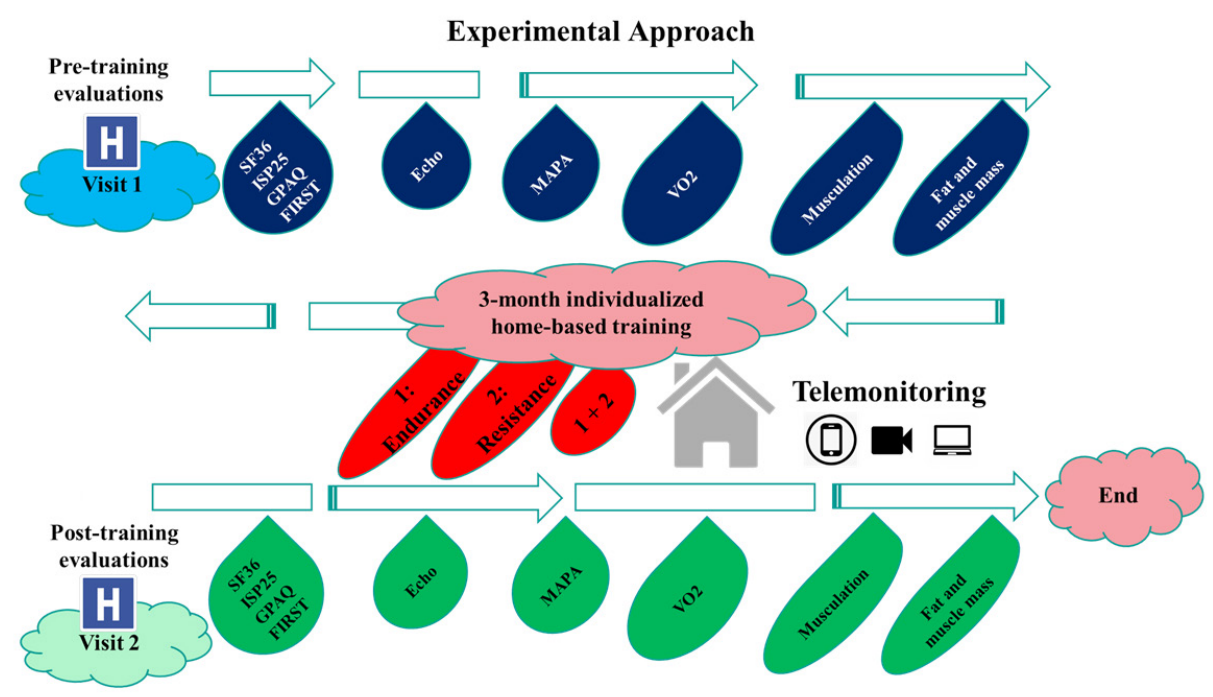

Figure 1. Illustration of the experimental approach. 
Table 1. Description of the endurance training circuit

\begin{tabular}{|c|c|c|c|c|c|}
\hline Time $\times$ number se-ries & Total time & Exercise time & Recovery time & Number of workshops & RPE scale \\
\hline $15^{\prime \prime}-15^{\prime \prime} \times 1$ & $5^{\prime}$ à $10^{\prime}$ & $15^{\prime \prime}$ & $15^{\prime \prime}$ & $3-6$ & $3 / 10$ \\
\hline $15^{\prime \prime}-10^{\prime \prime} \times 1$ & $5^{\prime}$ à $10^{\prime}$ & $15^{\prime \prime}$ & $10^{\prime \prime}$ & $3-6$ & $4 / 10$ \\
\hline $15^{\prime \prime}-5 " \times 1$ & $5^{\prime}$ à $10^{\prime}$ & $15^{\prime \prime}$ & $5^{\prime \prime}$ & $3-6$ & $5 / 10$ \\
\hline $20^{\prime \prime}-10^{\prime} \times 1$ & $5^{\prime}$ à $10^{\prime}$ & $20^{\prime \prime}$ & $10^{\prime \prime}$ & $3-6$ & $5 / 10$ \\
\hline $20 "-5^{\prime \prime} \times 1$ & $5^{\prime}$ à $10^{\prime}$ & $20 "$ & $5^{\prime \prime}$ & $3-6$ & $6 / 10$ \\
\hline $30 "-15^{\prime \prime} \times 1$ & $5^{\prime}$ à $10^{\prime}$ & $30 "$ & $15^{\prime \prime}$ & $3-6$ & $6 / 10$ \\
\hline $30 "-10^{\prime \prime} \times 1$ & $5^{\prime}$ à $10^{\prime}$ & $30 "$ & $10^{\prime \prime}$ & $3-6$ & $7 / 10$ \\
\hline $45^{\prime \prime}-10 " \times 1$ & $5^{\prime}$ à $10^{\prime}$ & $45^{\prime \prime}$ & $10^{\prime \prime}$ & $3-6$ & $8 / 10$ \\
\hline $50 "-10^{\prime \prime} \times 1$ & $5^{\prime}$ à $10^{\prime}$ & $50^{\prime \prime}$ & $10^{\prime \prime}$ & $3-6$ & $8 / 10$ \\
\hline
\end{tabular}

RPE: rating of perceived exertion.

Table 2. Description of the resistance training circuit

\begin{tabular}{|c|c|c|c|c|c|}
\hline Time $\times$ number se-ries & Total time & Exercise time & Recovery time & Number of workshops & RPE scale \\
\hline $20 "-30 " \times 1$ & $5^{\prime}$ à $10^{\prime}$ & $20 " / 8 \mathrm{r}$ & $<30^{\prime \prime}$ or more & $3-6$ & $3 / 10$ \\
\hline $20 "-30 " \times 1$ & $5^{\prime}$ à $10^{\prime}$ & $20 " / 8 \mathrm{r}$ & $<30^{\prime \prime}$ or more & $3-6$ & $4 / 10$ \\
\hline $20^{\prime \prime}-1^{\prime} \times 1$ & 5' à 10' & $20 " / 8 \mathrm{r}$ & $<1^{\prime}$ or more & $3-6$ & $5 / 10$ \\
\hline $20^{\prime \prime}-3^{\prime} \times 1$ & $5^{\prime}$ à $10^{\prime}$ & $20 " / 8 \mathrm{r}$ & $<3^{\prime}$ or more & $3-6$ & $5 / 10$ \\
\hline $30^{\prime \prime}-3^{\prime} \times 1$ & $5^{\prime}$ à $10^{\prime}$ & $30 " / 8 \mathrm{r}$ & $<3^{\prime}$ or more & $3-6$ & $7 / 10$ \\
\hline $30^{\prime \prime}-3^{\prime} \times 1$ & $5^{\prime}$ à $10^{\prime}$ & $50 " / 12 \mathrm{r}$ & $<3^{\prime}$ or more & $3-6$ & $7 / 10$ \\
\hline
\end{tabular}

RPE: rating of perceived exertion.

patients. Globally, effort times were 15 to 50 seconds interspersed with recovery phases of 15 to 50 seconds and the series duration was 3 to 12 minutes. The training session time was 60 minutes. Exercises were performed at bodyweight or with low additional loads ( $20 \%$ of the $1 \mathrm{RM}$ maximum).

\subsubsection{Resistance training (Group 3)}

The resistance training consisted of a TC composed of a sum of series each comprising two times: 1) "effort" and 2) "recovery" (Table 2). Each series included a succession of dynamic or static exercises, chosen individually according to the initial assessment, and a rest time. The number of repetitions during the effort was between 6 and 12. The recovery times were higher than the effort time from 30 seconds to 3 minutes. The total duration of the workout was 60 minutes. All muscles were solicited in both specific and exclusive work (i.e. lower limb type, abdominal belt, or upper limb) or combined (i.e. grouping together several muscle groups). Initially, patients carried out work of coordination of gestures specific to muscular reinforcement. Then, they performed muscle-building sessions to body weight. Finally, the various exercises were performed with a higher load that can range from $30 \%$ to $50 \%$ of the $1 \mathrm{RM}$ depending on the capabilities of the patients.

2.8.3. Combination of endurance and resistance training (Group 4)

This training program consisted of 2 sessions alternating 1 endurance TC session and 1 resistance TC session per week. The session of endurance was performed at a moderate-intensity corresponding to the target HR of VT1 and lasted 60 minutes. The session of resistance was performed at the HR of VT1, lasted 60 minutes and integrated muscular loads corresponding to $30-50 \%$ of the $1 \mathrm{RM}$.

\subsection{Telemonitoring}

The investigators followed the training program weekly based on phone interviews and vidioconferences. Patients had a cardiofrequencemeter and a tensiometer before beginning the training at home. During each session of exercise, HR, DBP, SBP and the RPE scale were monitored. Patients were informed at visit 1 of the criteria for alarm at rest and for stopping exercise. These criteria were: at rest: a SBP of $>140 \mathrm{mmHg}$ and a DBP of $>90 \mathrm{mmHg}$; on exertion: the SBP must remain $\leq 160$ $\mathrm{mmHg})(12,14-16)$. Patients also had a training booklet made by the investigators at their disposal to understand the exercises to be done.

Depending on variations of these parameters as training progresses, the investigators adjusted the exercise time and the recovery time in order to vary the intensity of the training. Moreover, a mismatch between the target training HR (i.e. HRVT1) and the HR communicated by the patient caused an adaptation of the work intensity, if necessary.

\subsection{Statistical analyses}

The ANOVA between the trained patients and the control patients was performed. Then the data of the 3 trained 
subgroups was compared by ANOVA. Qualitative data were reported as a percentage, and quantitative data as means and standard deviation. All results were expressed as mean \pm SD. The Software used was SPSS 20. Differences were considered significant at $P$ values $\leq$ 0.05 .

\section{Results and Discussion}

Regular physical activity is highly beneficial for patients with cardiovascular disease. Indeed, physically active patients have a decrease in cardiovascular mortality and in the risk of increasing the severity of their pathology (22). Moreover, regular, moderate to intense exercises have been reported to significantly lower blood pressure and therefore may have a significant impact on the appearance of cardiovascular diseases and aorthopathy (23-25). Patients with MFS might also benefit from regular physical activity but, to date, there are no randomized studies evaluating the longitudinal effect of exercise on survival or risk of aortic dissection in patients with aorthopathy including MFS patients (26).

Only two studies performed on a mouse model of MFS demonstrated that a five-month training consisting of moderate to intense dynamic exercises induced either an improvement of the aortic wall elasticity (27) or no significant reduction of the aortic diameter (28) compared to sedentary control animals. Only one study performed in patients with MFS demonstrated the feasibility of training in such patients and no adverse events were found after the three-week training program during their one-year follow-up (11). Hence, there still is no evidence of contraindications for an exercise prescription in patients with MFS. Moreover, there is a need to investigate the precise effects of training in such patients has been recently mentioned $(10,26)$. In this context, the preliminary results of our study that is underway are very encouraging. At this time, 8 patients (means: age: $30 \pm 2$ years; height: $178 \pm 2 \mathrm{~cm}$; weight: $66 \pm 6$ $\mathrm{kg}$ ) completed the 3-month combined training protocol. There is a tendency towards an increase of the score for their QoL between pre- and post-training (74 $\pm 15 v s$. $91 \pm 6 \approx+25 \%$ ). The evolution of their physiological parameters post-training also showed encouraging results. The VO2peak increased from $24 \pm 4$ to $27 \pm 5$ $\mathrm{mL} / \mathrm{min} / \mathrm{kg}(p=0.05)$, the slope of $\mathrm{VE} / \mathrm{VCO}_{2}$ tended to decrease from $34 \pm 19$ to $24 \pm 2$, the pulsed wave speed decreased from $5.3 \pm 0.4$ to $5.1 \pm 0.4 \mathrm{~ms}(p<0.001)$, the Aix decreased from $24 \pm 12$ to $16 \pm 13(p<0.001)$, the FEVG tended to increase from $60 \pm 11$ to $64 \pm 12 \%$, and the $1 \mathrm{RM}$ increased from $64 \pm 29$ to $96 \pm 25 \mathrm{~kg}(p<0.05)$. Interestingly, despite the moderate intensity of training, the aortic diameter did not change between pre and post training $(39.0 \pm 52 v s .39 .3 \pm 4.6 \mathrm{~mm})$. This potentially demonstrates the safety of the training program and its feasibility for Marfan patients. We think that the other results from the whole population study will follow the previously described results and permit us to propose a new healthcare for MFS patients in the future based on adapted physical activity and telemonitoring.

Telemonitoring is a unique feature of the training, facilitating control and compliance of the performed physical exercises by patients at home. In a review comparing studies performing telemonitoring or a traditional rehabilitation program in patients with cardiovascular diseases, authors reported that telemonitoring was as relevant as traditional training to induce benefits with no adverse effects in such patients (29). Again, based on our preliminary results, it is believed that patients with MFS might also benefit from an adapted home-based training program.

In conclusion, this personalized home-based training program appears to improve the QoL of MFS patients. It is anticipated that this specific training program associating mild to moderate regular endurance and resistance exercises should be prescribed because of its known positive effects on global health. It is also believed that this study will prove that telerehabilitation is a safe intervention and that it can help to prevent thoracic aortic dissection by implementing it into standard preventive care for patients with MFS.

\section{Acknowledgements}

We thank all study patients for their participation during the training sessions at home.

Funding: This study was supported by the FavaMulti - Rare diseases grant, the Casden prize (Fondation de l'Avenir) and the Association Marfan prize.

Conflict of Interest: The authors have no conflicts of interest to disclose.

\section{References}

1. Abd El Rahman M, Haase D, Rentzsch A, Olchvary J, Schäfers HJ, Henn W, Wagenpfeil S, Abdul-Khaliq H. Left ventricular systolic dysfunction in asymptomatic Marfan syndrome patients is related to the severity of gene mutation: Insights from the novel three dimensional speckle tracking echocardiography. PLoS One. 2015; 10:e0124112.

2. Alpendurada F, Wong J, Kiotsekoglou A, Banya W, Child A, Prasad SK, Pennell DJ, Mohiaddin RH. Evidence for Marfan cardiomyopathy. Eur J Heart Fail. 2010; 12:10851091.

3. de Witte P, Aalberts JJ, Radonic T, Timmermans J, Scholte AJ, Zwinderman AH, Mulder BJ, Groenink M, van den Berg MP. Intrinsic biventricular dysfunction in Marfan syndrome. Heart. 2011; 97:2063-2068.

4. Behan WM, Longman C, Petty RK, Comeglio P, Child AH, Boxer M, Foskett P, Harriman DG. Muscle fibrillin deficiency in Marfan's syndrome myopathy. J Neurol Neurosurg Psychiatry. 2003; 74:633-638.

5. Percheron G, Fayet G, Ningler T, Le Parc JM, Denot- 
Ledunois S, Leroy M, Raffestin B, Jondeau G. Muscle strength and body composition in adult women with Marfan syndrome. Rheumatology (Oxford). 2007; 46:957962.

6. Giske L, Stanghelle JK, Rand-Hendrikssen S, Strøm V, Wilhelmsen JE, Røe C. Pulmonary function, working capacity and strength in young adults with Marfan syndrome. J Rehabil Med. 2003; 35:221-228.

7. Benke K, Ágg B, Pólos M, Sayour AA, Radovits T, Bartha E, Nagy P, Rákóczi B, Koller Á, Szokolai V, Hedberg J, Merkely B, Nagy ZB, Szabolcs Z. The effects of acute and elective cardiac surgery on the anxiety traits of patients with Marfan syndrome. BMC Psychiatry. 2017; 17:253.

8. Peters KF, Kong F, Horne R, Francomano CA, Biesecker BB. Living with Marfan syndrome I. Perceptions of the condition. Clin Genet. 2001; 60:273-282.

9. Erbel R, Aboyans V, Boileau C, et al. 2014 ESC Guidelines on the diagnosis and treatment of aortic diseases. Kardiol Pol. 2014; 72:1169-1252. (in Polish)

10. Pelliccia A, Sharma S, Gati S, et al. 2020 ESC Guidelines on Sports Cardiology and Exercise in Patients with Cardiovascular Disease. Rev Esp Cardiol (Engl Ed). 2021; 74:545.

11. Benninghoven D, Hamann D, von Kodolitsch Y, Rybczynski M, Lechinger J, Schroeder F, Vogler M, Hoberg E. Inpatient rehabilitation for adult patients with Marfan syndrome: an observational pilot study. Orphanet J Rare Dis. 2017; 12:127.

12. Batalik L, Filakova K, Batalikova K, Dosbaba F. Remotely monitored telerehabilitation for cardiac patients: A review of the current situation. World J Clin Cases. 2020; 8:18181831.

13. Cheng A, Owens D. Marfan syndrome, inherited aortopathies and exercise: what is the right answer? Heart. 2015; 101:752-757.

14. Douard H. Nouvelles recommandations pour la pratique du sport de compétition en cas d'anomalie cardiovasculaire. Arch Mal Coeur Vaiss Prat. 2016; 2016:27-30. (in French)

15. Vorp DA, Schiro BJ, Ehrlich MP, Juvonen TS, Ergin MA, Griffith BP. Effect of aneurysm on the tensile strength and biomechanical behavior of the ascending thoracic aorta. Ann Thorac Surg. 2003; 75:1210-1214.

16. Foster C, Florhaug JA, Franklin J, Gottschall L, Hrovatin LA, Parker S, Doleshal P, Dodge C. A new approach to monitoring exercise training. J Strength Cond Res. 2001; 15:109-115.

17. Ware JE Jr, Sherbourne CD. The MOS 36-item short-form health survey (SF-36). I. Conceptual framework and item selection. Med Care. 1992; 30:473-483.

18. Bull FC, Maslin TS, Armstrong T. Global physical activity questionnaire (GPAQ): nine country reliability and validity study. J Phys Act Health. 2009; 6:790-804.

19. Perrot S, Bouhassira D, Fermanian J; CEDR (Cercle d'Etude de la Douleur en Rhumatologie). Development and validation of the Fibromyalgia Rapid Screening Tool (FiRST). Pain. 2010; 150:250-256.
20. Ninot G, Delignieres D, Fortes M. L'évaluation de l'estime de soi dans le domaine corporel. Staps. 2000; 53:35-48.

21. Braverman AC, Harris KM, Kovacs RJ, Maron BJ. Eligibility and disqualification recommendations for competitive athletes with cardiovascular abnormalities: task force 7: aortic diseases, including Marfan syndrome: A scientific statement from the American Heart Association and American College of Cardiology. J Am Coll Cardiol. 2015; 66:2398-2405.

22. Nystoriak MA, Bhatnagar A. Cardiovascular effects and benefits of exercise. Front Cardiovasc Med. 2018; 5:135.

23. Fagard RH. Exercise characteristics and the blood pressure response to dynamic physical training. Med Sci Sports Exerc. 2001; 33:S484-92; discussion S493-4.

24. Fagard RH. Exercise is good for your blood pressure: effects of endurance training and resistance training. Clin Exp Pharmacol Physiol. 2006; 33:853-856.

25. Hardy ST, Loehr LR, Butler KR, Chakladar S, Chang PP, Folsom AR, Heiss G, MacLehose RF, Matsushita $\mathrm{K}$, Avery CL. Reducing the blood pressure-related burden of cardiovascular disease: Impact of achievable improvements in blood pressure prevention and control. J Am Heart Assoc. 2015; 4:e002276.

26. Thijssen CGE, Bons LR, Gökalp AL, Van Kimmenade RRJ, Mokhles MM, Pelliccia A, Takkenberg JJM, RoosHesselink JW. Exercise and sports participation in patients with thoracic aortic disease: a review. Expert Rev Cardiovasc Ther. 2019; 17:251-266.

27. Gibson C, Nielsen C, Alex R, Cooper K, Farney M, Gaufin D, Cui JZ, van Breemen C, Broderick TL, VallejoElias J, Esfandiarei M. Mild aerobic exercise blocks elastin fiber fragmentation and aortic dilatation in a mouse model of Marfan syndrome associated aortic aneurysm. J Appl Physiol (1985). 2017; 123:147-160.

28. Mas-Stachurska A, Siegert AM, Batlle M, Gorbenko Del Blanco D, Meirelles T, Rubies C, Bonorino F, SerraPeinado C, Bijnens B, Baudin J, Sitges M, Mont L, Guasch E, Egea G. Cardiovascular benefits of moderate exercise training in Marfan syndrome: Insights from an animal model. J Am Heart Assoc. 2017; 6:e06438.

29. Chan C, Yamabayashi C, Syed N, Kirkham A, Camp PG. Exercise telemonitoring and telerehabilitation compared with traditional cardiac and pulmonary rehabilitation: A systematic review and meta-analysis. Physiother Can. 2016; 68:242-251.

Received May 18, 2021; Revised July 29, 2021; Accepted August 25, 2021.

\section{*Address correspondence to:}

Damien Vitiello, URP 3625-Institute of Sport and Health Sciences of Paris (I3SP), Université de Paris, Paris 75015, France.

E-mail: damien.vitiello@u-paris.fr

Released online in J-STAGE as advance publication September 16, 2021. 